\title{
Practice Schedule And The Learning Of Motor Skills In Children And Adults: Teaching Implications
}

Genevieve Pinto Zipp, Seton Hall University, USA

A. M. Gentile, Teachers College/Columbia University, USA

\begin{abstract}
Understanding how motor skills are learned influences how one teaches effective motor skill attainment. Educators must ask, "Does repetitive practice of the same task make for better performance or does contextual variability (random practice) offer some benefit when learning motor skills?" Studies on the effects of Contextual Interference may provide some insight. Contextual interference (CI) studies typically use simple tasks involving movements already acquired by adults, which may account for random practice benefits. In contrast, children do not consistently demonstrate CI effects, as tasks usually require acquisition of a new movement pattern. In this experiment, adults and children ((8-10 yrs old) threw a Frisbee to targets for 54 trials under random or blocked conditions. Having had considerable throwing experience with other throwing objects, adults were expected to benefit from random practice. For children, a blocked practice advantage was predicted as it provides for devising and stabilizing a suitable movement pattern. Retention/retraining trials were administered 30 minutes after acquisition and seven days later. Two transfer tests were given after the delayed retention test: (a) same throwing object/different target distances (SODT), which required re-scaling the practiced movement, and (b) different throwing object (ropeball)/same target distances (DOST) which required a new movement pattern. Throwing accuracy was measured by absolute error from the target. Both age groups showed a blocked practice benefit on retention and SODT transfer tests. Findings from this experiment suggest that task variables and stage of learning are important determinants of CI effects and thus should influence how we teach motor skill attainment.
\end{abstract}

Keywords: contextual interference, skill acquisition, learning stages, transfer, retention

\section{INTRODUCTION}

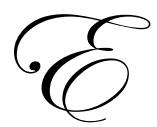

ducators who teach students how to effectively perform motor skills ranging from performing a sport, to playing an instrument, to manipulating a keyboard or to using a medical surgical simulator look to the literature to assist them in designing the most effective and efficient strategies for motor skill learning. In order to promote learning educators must ask, "Does repetitive practice of the same motor task make for better learning of the task or does randomly practicing different motor tasks demonstrate a greater benefit?"

When several variations of a movement task are learned concurrently, the question arises as to how practice should be structured. One variation of the task could be practiced for several trials before switching to another (blocked practice). Alternatively, task conditions could be varied over successive trials (random practice). It might be assumed that interference produced by repeatedly changing the practice context would be detrimental to learning. However, research contradicts this assumption (c.f., Magill and Hall, 1990; Brady, 1998). Although performance during acquisition may be impaired, random practice is often found to be better than blocked when learning is evaluated by tests of retention or transfer. This paradoxical pattern of findings is referred to as the contextual interference $(\mathrm{CI})$ effect. 
The CI effect usually found with adults is not consistently demonstrated with children. In several studies, no advantage of random over blocked practice was observed when children served as subjects (Del Rey, Whitehurst \& Wood, 1983; Pigott \& Shapiro, 1984; Wulf, 1991). Indeed, Del Rey et al. (1983) demonstrated otherwise: blocked superior to random practice on transfer tests. Similarly, Farrow and Maschette (1997) reported that younger children (8- to 9-year-olds) benefited most from blocked practice; only older children (10- to 12-year-olds) showed a random practice advantage. Although random, blocked or constant practice did not differ in the Pigott and Shapiro study (1984), a modified blocked schedule (three successive trials under one condition before switching to another) was found to enhance children's learning.

Practice schedules may affect children differently than adults because of two factors: task variables and prior experience. Most CI studies with adults have used laboratory tasks involving simple movements, such as moving the hand from one location to another to knock down a barrier or depress a key (Shea \& Morgan, 1979). Often, what is learned in such tasks is to perform these previously acquired movements in a particular order or temporal sequence. Although not initially proficient in these laboratory tasks, adults readily organize the required pattern and seem to progress quickly to a more advanced stage of learning. It may be that CI effects with adults occur because random practice is advantageous only during later phases of learning. Support for this possibility comes from the findings of Shea, Kohl and Indermill (1990) where random practice on a rapid force production task enhanced learning only after 200 training trials. During initial practice (the first 50 trials), blocked was better.

Unlike the simple laboratory tasks used with adults, most CI studies with children have used complex multijoint or whole body movements, such as beanbag throws to targets (Wulf, 1991) or forehand tennis strokes (Farrow \& Maschette, 1997). With limited prior experience, young children probably are at an early stage of learning such complex movements and, thus, may not profit from random practice. During initial practice of complex tasks, the performer has to organize a new movement form or topology that is structured to fit the environmental context (Bernstein, 1967; Gentile, 1972). As the novice performer tries to assemble a suitable pattern, multijoint movement is characterized by high intrinsic variability. Novices lack precise control of intersegmental dynamics so that reactive forces and peripheral field effects are not accounted for in advance and perturb the movement's flow (c.f. Gentile, 2000). Adding extrinsic variability by frequently changing task conditions may impede stabilizing an appropriate movement form. We hypothesized that the movement's topology must be resolved before the performer can cope effectively with highly variable conditions (random practice). This proposal implies that novice performers would benefit by restricting variability in task conditions. Therefore, blocked practice was predicted to enhance learning more than random during initial practice of complex, whole-body movements by the young novice children in our study.

Contrary to our predictions, Pollock and Lee (1997) have reported that random practice benefited both children and adults. Their task required seated subjects to use a rapid single-joint movement of one finger to propel a small ball to one of three tabletop targets. Apparently, children and adults learned to scale the magnitude and direction of force for this simple movement, which was already available in their repertoire. Whether children and adults would demonstrate the same CI effects when acquiring a novel whole-body movement was the question addressed in the present study.

Practice schedule were evaluated in children and adults during acquisition of a task involving a Frisbee throw to three target distances. The first objective was to compare practice effects for the two age groups and not to determine whether one practice condition facilitated transfer to the other. Therefore, retention tests were administered under the same conditions as during training. With limited familiarity with throwing objects shaped like a Frisbee, children were expected to need considerable practice to develop a movement topology appropriate for the task. Thus, children were assumed to be at an early phase of learning and were expected to demonstrate an advantage of blocked over random practice. Although novices at the task, adults were expected to utilize prior throwing experiences to organize an effective movement form and quickly progress to a later phase of learning. Thus, it was hypothesized that adults would show a random practice benefit.

The second objective was to evaluate transfer to two task variations that were administered using a random order: (a) Frisbee throw to targets located nearer and farther than those used during training (Same Object/Different 
Target), and (b) ropeball throw to the same targets practiced during acquisition (Different Object/Same Targets). The Same Object/Different Target test required setting new movement parameters while maintaining a similar typology for the Frisbee throw. For children, acquisition of the movement typology was expected to be poorer after random than blocked practice. Thus, on Same Object/Different Target task, re-scaling the movement form was expected to be easier after blocked practice, even though testing involved a random order. As adults were expected to rapidly acquire the movement form during practice the opposite pattern was predicted, random better than blocked. The second transfer task, Different Object/Same Targets, involved a new movement pattern requiring a change in typology and motor parameters. The inclusion of this second test enabled us to evaluate whether simply familiarity and attunement to target locations provided for transfer effects.

\section{METHOD}

\section{Participants}

Twelve adults (6 males, 6 females) $20-30$ yrs old $(M=26.1)$ volunteered to participate. Twelve children (6 boys, 6 girls), 8-10 yrs of age ( $M=9.6)$ assented to participate with informed consent given by legal guardians. To insure that participants were novices in the task, they were pre-tested on five trials of throwing a Frisbee at a single target. To be included in the study, subjects had to have fewer than three throws within the defined target area. Adults and children were randomly assigned to either blocked or random practice conditions. Thus, there were four experimental groups; Child/Blocked, Child/Random, Adult/Blocked, Adult/Random ( $\mathrm{n}=6$ per group).

\section{Tasks}

Two tasks were used: Frisbee throw and ropeball toss. The tasks involved throwing a Frisbee (diameter: $17.46 \mathrm{~cm}$ ) or ropeball (diameter: $17.78 \mathrm{~cm}$, hollow center, flexible outer rope), with the preferred hand, to a target that was designated by a specific color. Acquisition targets were "red" (near distance), "yellow" (middle) and "blue" (far); and the transfer test targets were "green" (nearer) and "orange" (farther). Subjects were instructed that the goal of the task was to have the Frisbee or ropeball land entirely within the verbally specified, colored area of the target. No instruction was given concerning how the movement should be organized. A starting square $(50.80 \mathrm{~cm}$ in diameter) was constructed from a green rubber mat, attached to the floor by masking tape along its outer rim. Participants had to maintain their standing position within the starting square while throwing the object to the 3 targets which were positioned at distances involving $91.44 \mathrm{~cm}$ increments, measured from the center of a starting square to the target's center (the first target was positioned $182.88 \mathrm{~cm}$ from the starting square). During the SODT transfer test, location of targets from the starting square was $91.44 \mathrm{~cm}$ (green: nearer target) and $457.2 \mathrm{~cm}$ (orange: farther target).

\section{Procedure}

During acquisition under blocked practice, subjects threw the Frisbee to each of the 3 targets for 9 consecutive trials repeating the blocked format again for a total of 54 trials (order counterbalanced across subjects within each age group). For random practice, each of the 3 targets was presented 9 times in a random order within each of two blocks of 27 trials (total trials $=54$ trials), with the restriction that a given target was tested no more than twice in succession. All subjects received a 3 min rest period between blocks of trials. During these rest periods, subjects were given age-appropriate books to read and questions to answer pertaining to these readings to ensure that implicit practice of the task did not occur.

Retention trials followed the same testing procedures as during acquisition. For blocked practice, 9 successive trials at each of the 3 targets were tested (total $=27$ trials) with order counterbalanced across subjects. For random practice, 9 test trials at each of the 3 targets were completed in random order. There were two retention tests: (a) immediate: $30 \mathrm{~min}$ following acquisition; and (b) delayed: 7 days later. During the $30 \mathrm{~min}$ interval prior to the immediate test, participants engaged in activities similar to those used in rest periods. Participants were instructed not to engage in any Frisbee throwing activities during the retention interval. Two transfer tasks were administered following the delayed retention test: (a) Same Object/Different Targets (SODT) that involved throwing the Frisbee to 
targets nearer and farther than those used during acquisition, (b) Different Object/Same Targets (DOST) that involved throwing the ropeball to the same three acquisition targets. Order of testing these two transfer tasks was counterbalanced across subjects. For both transfer conditions, four test trials on each target were administered using a random order. Throwing accuracy was measured in terms of absolute error of object from target. Deviation from the center of the target to the center of the Frisbee or to the edge of the ropeball was measured in centimeters.

\section{Design}

Multifactor analysis of variance (ANOVA) was carried out for each phase of the experiment: acquisition, retention/retraining and transfer. Generally, the design was a 2 (Practice: random vs. blocked) x 2 (Age: children vs. adults) between-groups factorial with another factor added, when relevant (e.g., blocks of acquisition trials). Partial Eta squared (eta sqr) was the measure of effect size. Scheffe post hoc analysis ( $\mathrm{p}<$. 05) was used for individual comparisons between means.

\section{RESULTS}

\section{Acquisition}

Children displayed poorer throwing accuracy than adults but performance improved over blocks of trials for both age groups. The ANOVA of error scores indicated main effects of Age, F $(1,20)=12.29$, p<.002, eta sqr=. 38, and Trial-Blocks, $\mathrm{F}(5,20)=3.44$, p<. 007, eta sqr=. 15. Practice and interaction effects were not significant. As shown in Figure 1, mean error tended to be higher for the random than block groups (overall means: random = 51.75 ; block $=38.40)$. However, there was only a weak suggestion that this difference was reliable, $\mathrm{F}(1,20)=2.95$, $\mathrm{p}<.10$, eta $\mathrm{sqr}=.13$, which was probably due to the high variability within groups resulting in limited statistical power (.37).

\section{Retention}

During retention tests, throwing accuracy was better following blocked practice than random practice for both children and adults. The ANOVA yielded main effects of Practice, $\mathrm{F}(1,20)=4.29$, p<. 05, eta sqr=. 18, and Age, $\mathrm{F}(1,20)=42.69$, p<. 001, eta sqr=. 68. Other effects were not statistically reliable.

\section{Transfer Tests}

On the SODT transfer test, children trained under random conditions performed notably poorer than all other groups. The ANOVA indicated a main effect of Age, F $(1,20)=5.30, \mathrm{p}<$. 03, eta sqr=. 21, and a strong suggestion of an Age x Practice interaction, $F(1,20)=3.88, \mathrm{p}<.06$, eta sqr $=.16$. Individual comparisons between means showed comparable throwing accuracy on SODT for children and adults trained with blocked practice. In contrast, random practice for children produced significantly poorer performance than adults.

On the DOST transfer test, the only finding was that adults had better throwing accuracy than children. The Age effect was statistically.

\section{CONCLUSION}

In this Experiment, throwing accuracy improved during acquisition for both age groups with no evidence of a difference between random and blocked conditions. However, as predicted, retention was better after blocked practice than random practice, which is contrary to the CI effects typically reported (Jarus \& Goverover, 1999). Both adults and children showed a blocked advantage on retention tests possible because both groups were still in the early stage of learning the task as a possible result of the low number of acquisition trials (54) and thus benefited from the repetitive nature associate with blocked practice. These findings lend support to our proposal that restricting task variability during initial learning of complex tasks is beneficial because it provides an opportunity for the learner to stabilize their movement topology as a result of its repetitiveness. 
On the SODT transfer test, two findings are noteworthy. First, children and adults trained under blocked practice performed at similar levels. Thus, the age difference in throwing accuracy evident throughout acquisition was no longer present. Evidently, blocked practice so enhanced children's learning that they were able to cope with the new Frisbee targets similarly to that of the adult findings. Second, children trained under random practice showed the poorest transfer on the SODT test. This finding, in combination with the retention data, confirmed our proposal that children do not benefit from random practice during initial learning of complex multijoint movement tasks. On the DOST transfer test, adults performed better than children with no effect of practice conditions. The DOST test required a movement pattern that differed in topology and motor parameters from the Frisbee throw. The adult's prior experiences in throwing diverse objects apparently enabled them to deal with these new task-demands more successfully than children.

\section{DISCUSSION}

Learning was not enhanced by high task-variability during initial practice of a complex, whole-body movement by novice performers. The retention findings for both children and adults as well as the transfer data for children indicated that blocked practice benefited initial learning more than random. We had hypothesized that performers must derive a general solution to the motor problem during early learning before they are able to cope effectively with contextual variability. It was assumed that a novice performer must first discover how to map body morphology to environmental constraints that, using Green's metaphor (1972), "gets into the ballpark" to accomplish the task-goal. In complex movements, such as the Frisbee throw in the present experiments, arriving at a suitable movement topology or general movement form is no easy task. During initial learning of multijoint whole-body movements, control of intersegmental dynamics is poor (Gentile, 2000). Failure to predicatively account for reactive forces and linkage effects results in perturbations making the movement intrinsically variable. In addition, temporal coordination of simultaneous and successive movement components has not been refined. Adding extrinsic variability during early learning, by using random practice, apparently hinders development of an appropriate movement topology as reflected by poorer throwing accuracy in the present experiments. In the present study, children were assumed to have less experience in throwing objects, especially one shaped like the Frisbee. With less positive transfer, they were proposed to be at an early stage of learning. To stabilize an appropriate movement topology, children were expected to benefit from less variability in task-conditions. The findings for children on the retention tests supported these proposals: blocked practiced enhanced learning more than random.

The benefits of restricting task-variability during early learning of whole-body movements are not limited to children. Adults who are novices in such tasks also profit by consistent task-conditions. When adults were limited to an initial learning phase by the limited number of acquisition trials, blocked practice yielded better retention than random practice. As suggested by Hebert, Landin and Solomon (1996), whole-body movements are more complex than typical laboratory-tasks because a greater number of degrees of freedom must be controlled. They proposed that some proficiency in the task is necessary before novices benefit from a random schedule. In learning tennis strokes, they found that novice low-skill subjects showed a blocked practice advantage. In contrast, no difference was evident between an alternating and blocked schedule for skilled subjects. However, given the Power Law of learning (Gentile, 1998), the extent of practice (30 strokes during each of 9 sessions) may have been inadequate to produce a significant performance increment for the skilled subjects. In contrast, Guadagnoli, Holcomb and Weber (1999) reported typical CI findings for skilled but not unskilled performers during acquisition of a whole-body movement in a putting task. Skilled putters, assumed to be at an advanced phase of learning, demonstrated a random practice advantage. The novice putters profited by a blocked schedule.

Others have proposed that laboratory-based studies should not be generalized to teaching strategies in reallife, sport situations (Hoffman, 1990; Brady, 1998). Indeed, when practice schedules have been examined using sport activities in field-based studies, findings have been inconclusive or quite mixed (Bortoli, Robazza, Durigon \& Carra, 1991; French, Rick \& Werner, 1990; Good \& Magill, 1986, Sears \& Husak, 1987). Findings of the present study support their argument.

We propose that the inherent complexity of motor control in multijoint, whole-body movements is the critical factor limiting generalizability of findings from simple laboratory tasks. Complexities associated with 
intersegmental dynamics and coordination of simultaneous and successive movement components pose little challenge to performers who already know how to move their hand from one location to another to knock down barriers or depress switches (common laboratory-type tasks). However, these coordination and control issues during initial learning of multijoint movements make development of a stable topology difficult when the performer is also confronted with high contextual variability.

During acquisition, reliable differences between random and blocked conditions were not evident in the present experiments. Supposedly, random practice induces high interference, which degrades performance during acquisition, whereas blocked, does not. Such interference is viewed as facilitory to learning as represented by the paradoxical pattern of findings on retention and transfer tests. Failure to demonstrate a difference between random and blocked practice is not uncommon; several studies have reported similar findings (Magill \& Hall, 1990, Brady 1998). In the current experiment, there was a suggestion, though not statistically reliable, that performance during acquisition was poorer under random than blocked practice. To the extent that this may have represented interference, it was not beneficial to learning, as shown by superior retention after blocked practice. Random practice may produce interference during initial practice of whole-body movements but this interference does not appear beneficial to learning.

In summary, the present experiment found that for young children and adults who are novices in performing whole-body movements, movement variability during initial practice is already high as the novice attempts to discover an appropriate topology and cope with the complexities of motor control. Adding contextual variability by using a random practice schedule was shown to be less profitable during early acquisition of tasks requiring wholebody movements. However, once beyond an initial learning stage, high contextual variability did benefit adult learning as it may have promoted tuning of the general movement solution to variations in task-conditions. Future studies need to assess the effects of practice organization at the movement level to support our proposals that the inherent complexity of motor control in multijoint, whole-body movements is the critical factor limiting generalizability of findings from simple laboratory tasks. Thus, for instructors of motor skills there is no easy answer as to how best to teach motor skills. When designing an environment to promote motor skill learning, instructors must consider the level of the learning (novice or experienced), complexity of the motor skill to be learned, the learners cognitive abilities and their ability to attend and use their critical thinking skills to continually assess the effects of the learning environment.

\section{AUTHOR INFORMATION}

G. Pinto Zipp PT, EdD is Associate Professor and Chair of the Department of Graduate Programs in Health Sciences at Seton Hall University. She received her EdD from Teachers College Columbia University in 1996. She currently teaches management of neuromuscular problems in the Doctor of Physical Therapy program. Her research interests focus on, a) effects of performing dual tasks on walking performance and postural sway in children and adults, and b) curriculum design issues including the use of mind mapping and video based cases in professional education for the promotion of clinical decision making skills.

A.M. Gentile $\mathrm{PhD}$ has been a renowned researcher in the field of motor learning and control for over 40 years and is the past chair of the Movement Science and Education Program Teachers College, Columbia University.

\section{REFERENCES}

1. Bernstein A. (1967). The coordination and regulation of movement. New York: Pergamon Press.

2. Bortoli, Robazza, Durigon, Carra. (1992). Effects of contextual interference on learning technical sport skills. Perceptual and Motor Skills, 75, 552-562.

3. Brady, F. (1998). A theoretical and empirical review of the contextual interference effect and the learning of motor skills. Quest, 50, 266-293.

4. Del Rey, P., Whitehurst, M. \& Wood, J. (1983). Effects of experience and contextual interference on learning and transfer by boys and girls. Perceptual and Motor Skills, 56(2), 581-582. 
5. Del Rey, P., Whitehurst, M., Wughalter, E., \& Barnwell, J. (1983). Contextual interference and experience in acquisition and transfer. Perceptual and Motor Skills, 57(1), 241-242.

6. Farrow, D., \& Maschette, W. (1997). The effects of contextual interference on children learning forehand tennis groundstrokes. Journal of Human Movement Studies, 33, 47-67.

7. French, K., Rick, J., \& Werner, P. (1990). Effects of contextual interference on retention of three volleyball skills. Perceptual and Motor Skills, 71, 179-186.

8. Gentile, A.M. (1972). A working model of skill acquisition with application to teaching. Quest Monograph, XVII, 3-23.

9. Gentile, A. M. (1998). Implicit and explicit processes during acquisition of functional skill. Scandinavian Journal of Occupational Therapy

10. Gentile, A.M. Skill acquisition: Action, movement, and neuromotor processes. In J. Carr \& R. Shepherd (Eds.) Movement Sciences: Foundation for Physical Therapy in Rehabilitation, $2^{\text {nd }}$ Ed. Gaithersburg, Maryland: Aspen Publication, 2000, pp. 111-188.

11. Green, P.H. Problems of organization of motor systems. Progress in Theoretical Biology. Vol.2 New York: Academic Press, 1972, pp. 303-338.

12. Guadagnoli, Holcomb, Weber, (1999). The relationship between contextual interference effects and performer expertise on learning of a putting task. Journal of Human Movement Studies, 37, 19-36.

13. Higgins, J.R., \& Spaeth, R.K. (1972). Relationship between consistency of movement and environmental condition. Quest, 17, 61-69.

14. Jarus, T. \& Goverover, Y. (1999). Effects of contextual interference and age on acquisition, retention, and transfer of motor skill. Perceptual and Motor Skills, 88, 437-447.

15. Jarus, T., Wughalter, E.H., \& Gianutsos, J.G. (1997). Effects of contextual interference and conditions of movement task on acquisition, retention, and transfer of motor skills by women. Perceptual and Motor Skills, 84, 179-193.

16. Jelsma, O., \& Van Merrienbou, J. (1989). Interactions with reflection-impulsivity. Perceptual and Motor Skills, 68, 1055-1064.

17. Lee, T.D., Wulf, G., \& Schmidt, R.A. (1992). Contextual interference in motor learning: Dissociated effects due to the nature of task variations. The Quarterly Journal of Experimental Psychology, 44A (4), 627-644.

18. Lee, T. D., Swanson, L. R., \& Hall, A. L. (1991). What is repeated in a repetition? Effects of practice conditions on motor skill acquisition. Physical Therapy: Movement Science Series, 71(2), 150-156.

19. Magill, R. A. \& Hall, K. G. (1990). A review of the contextual interference effect in motor skill acquisition. Human Movement Science, 9, 241-289.

20. Newell, K.M., \& Shapiro, D. C. (1976). Variability of practice and transfer of training: Some evidence toward a schema view of motor learning. Journal of Motor Behavior, 8(3), 233-243.

21. Pigott, R.E. \& Shapiro, D. C. (1984). Motor schema: The structure of the variability session. Research Quarterly for Exercise and Sport, 55, 41-45.

22. Pinto Zipp, G., \& Gentile, A. M. (1995). Practice schedule and motor learning: Children versus adults. Society for Neuroscience Abstract, $X$, 792.1).

23. Pollatou, E., Kioumourtzoglov, E., Agelousis, N., \& Mavromatis, G. (1997). Contextual interference effects in learning novel motor skills. Perceptual Motor Skills, 84, 487-496.

24. Pollock, B.J., \& Lee, T.L. (1997). Dissociated contextual interference effects in children and adults. Perceptual and Motor Skills, 84, 851-858.

25. Porretta, D. (1988). Contextual interference effects on the transfer and retention of a gross motor skill by mildly mentally handicapped children. Adapted Physical Activity Quarterly, 5, 332-339.

26. Proteau, L., Blandin, Y., Alain, C., \& Dorion, A. (1994). The effects of the amount and variability of practice on the learning of a multi-segmented motor task. Acta Psychologica, 85, 61-74.

27. Schmidt, R.A. (1975). A schema theory of discrete motor skill learning. Psychological Review, 82, 225260.

28. Schmidt, R.A. (1988). Motor control and learning: A behavioral emphasis. 2nd Ed. Champaign, Illinois: Human Kinetics Publishers, Inc.

29. Schmidt, R.A. (1991). Motor learning principles for physical therapy. In: Lister, M.J. (Ed). Contemporary Management of Motor Control Problems. Fredericksburg, Vr: Bookcrafters, Inc; 49-64. 
30. Sears, C. \& Husak, W.S., (1987, April 13-17). Contextual effect in an educational setting: An example of level three research. Paper presented at the National Convention of the American Alliance for Health, Physical Education, Recreation and Dance, Las Vagus, NV.

31. Shea, C. H., Kohl, R., \& Indermill, C. (1990). Contextual interference contributions of practice. Acta Psychologica, 73, 145-157.

32. Shea, J. B., \& Morgan, R. L. (1979). Contextual interference effects on the acquisition, retention, and transfer of a motor skill. Journal of Experimental Psychology: Human Learning and Memory, 5(2), 179187.

33. Shumway-Cook, A., \& Woollacott, M. (1985). The growth of stability: Postural control from a developmental perspective. Journal of Motor Behavior, 17, 131-147.

34. Tsutsui, S., Lee, D., \& Hodges, N. (1998). Contextual interference in learning new patterns of bimanual coordination. Journal of Motor Behavior, 30, 151-157.

35. Woollacott, M. H., Shumway-Cook, A. (Eds.). (1989). Development of Posture and Gait Across the Life Span. South Carolina: University of South Carolina Press.

36. Wrisberg, C. A (1991) A field test of the effects of contextual variety during skill acquisition. Journal of Teaching Physical Education, 11, 21-30.

37. Wulf, G. (1991). The effect of type of practice on motor learning in children. Applied Cognitive Psychology, 5, 123-134.

38. Wulf, G., \& Lee, T. D. (1993). Contextual interference in movements of the same class: Differential effects on program and parameter learning. Journal of Motor Behavior, 25(4), 254-263.

39. Wulf, G., \& Schmidt, R. A. (1988). Variability in practice: Facilitation in retention and transfer through schema formation or context effects? Journal of Motor Behavior, 20, 133-149.

40. Wulf, G., \& Schmidt, R. A. (1989). The learning of generalized motor programs: Reducing the relative frequency of knowledge of results enhances memory. Journal of Experimental Psychology: Learning, Memory, and Cognition, 15, 748-757. 